\title{
The Internet as a medium for delivering treatment for depression. Possibilities and challenges highlighted by our experiences with MoodGYM
}

\section{Nils Kolstrup}

Norwegian Centre for Integrated Care and Telemedicine and UiT The Arctic University of Norway, Tromsø

Nils.Kolstrup@telemed.no

Kolstrup, N (2014). The Internet as a medium for delivering treatment for depression. Possibilities and challenges highlighted by our experiences with MoodGYM. Tidsskrift for Forskning i Sygdom og Samfund, nr. 21, 19-36.

In this paper, I will present the current status of the use of the Internet as a medium for the treatment of depression. I will focus on the major challenges posed by the wide use of internet-based programmes. Recent and potential future developments of internet-based programmes for the treatment of depression will also be discussed.

I am a member of a research group at UiT The Arctic University of Norway (UiT). For the past seven years, we have been working with the internet-based depression-treatment programme MoodGYM. Our motivation to perform this research was partly based on local shortage of treatment capacity. These local problems are not confined to northern Norway. The world is presently experiencing a worldwide epidemic of depression with vast personal, social, and economic costs. Health-care systems strive to cope with these challenges and investigate whether internet-based programmes can help alleviate these problems with no or limited involvement of the health-care system. 


\section{Introduction}

I have been a member of a team working at the forefront of very exciting research involving psychology and computer technology. At UiT MoodGYM has been the subject of research since it was translated from English into Norwegian in 2006. Our research group ${ }^{1}$ has worked in close co-operation with the Centre for Mental Health Research at the Australian National University, Canberra, Australia. Our group has published several papers, which will be mentioned in their proper context.

Initially, studies were performed solely at the Institute of Psychology UiT. From 2011, studies have been conducted in co-operation with the General Practice Research Unit at the Department of Community Medicine in UiT.

The MoodGYM programme (https://moodgym.anu.edu.au/welcome) was developed by the Centre for Mental Health Research, Australian National University and can be used free of charge. Currently, it is translated into Norwegian, Dutch, and Mandarin. MoodGYM has 600,000 registered users.

MoodGYM was designed to be a training programme to prevent and, to some extent, to treat depression in young people between 15 and 25 years. However, an analysis of distribution of the age and sex of its users has shown that their average age is 35 years and that $60 \%$ are female (Christensen et al., 2002). The programme is based on cognitive behavioural therapy (CBT), interpersonal therapy, and relaxation therapy. It uses multimedia techniques with automated feedback from problem solving and self-evaluation in a dynamic fashion (Barak et al., 2009). The programme consists of five modules. Module 1 pertains to negative feelings and negative thoughts and how they interact. Module 2 describes, and lets the users practice, how thoughts will affect the way they feel about, understand, and react to different situations. Module 3 lets the users understand and practice challenging negative thoughts, and understand how these will affect their emotions. Module 4 illustrates how negative thoughts and stress interact, and lets the users practice relaxation techniques based on "mindfulness". Module 5 uses the principles from interpersonal therapy to help the users understand and use problemsolving techniques in their relations with friends and family. Several studies have shown that the programme is effective in reducing depression (Farrer et al., 2012; Powell et al., 2013) ${ }^{2 *}$ 


\section{The problem}

The World Health Organization has ranked depression as the fourth-leading cause of disability worldwide. It is projected that by 2020, depression will be the second-leading cause of human debility (Kessler \& Bromet, 2013). In industrial countries, depression causes the highest burden of disease, whereas in low-income countries, depression represents the third-highest burden of disease (Mathers \& Loncar, 2006). The economic costs of depression are huge and difficult to ascertain but vary greatly (Kleine-Budde et al., 2013; Sado et al., 2011). In 1990, there were an estimated 786,000 suicides in the world (Reza et al., 2001). According to the WHO, in Norway, the frequency of death by suicide is more than double that of death by traffic accidents (17.3 and 6.5 per 100,000 for males and females, respectively).

Depression is a mental stage with increasing negative focus, inactivity, and rumination as the symptoms become more pronounced (Mørch \& Rosenberg, 2005). In cases of severe depression, the patients are bedridden and cannot imagine a life after the depression. Major depressive disorders are associated with cognitive impairment. Episodic memory is affected and psychomotor activity, mental flexibility and attention are reduced. The function of working memory is hampered. For a thorough review, see Ebmeier et al. (2006). The biology and natural history of depression are poorly understood. Single episodes remit spontaneously in $45 \%$ to $71 \%$ of the patients after 12 months (Rhebergen et al., 2009; Wells et al., 1992). Fifty to sixty percent of patients have recurrent episodes of depression (Andrews, 2001).

However, depression can be treated. Psychotherapy is efficacious (Cuijpers et al., 2008b) and CBT is the most efficient therapy (Butler et al., 2006; Cuijpers et al., 2008b) on a level comparable to that of pharmacological treatment (Cuijpers et al., 2008a). Both medication and psychotherapy seem to be cost-effective (Prukkanone et al., 2012). But the cost of medication and the lack of trained health workers impede the treatment of depression (Collins et al., 2004; Andersson et al., 2013)*

\section{The Internet}

The Internet is widely available to the general population in industrialized countries and is spreading rapidly to low- and middle-income countries (http://www. Internetworldstats.com/stats.htm). Therefore, perhaps internet-based programmes could be used to change help-seeking behaviours and attitudes. The Internet could also increase self-help and improve treatment. 
The Internet is international and can reach areas that are remote and have very limited infrastructure. Currently, health information is available in remote areas of the world (Edejer, 2000; Godlee et al., 2004). Although little is known on the effect of large scale internet based interventions for depression, studies suggest that interventions for depression delivered in primary care are as cost-effective as anti-retroviral drugs for HIV/AIDS (Patel et al., 2007). To obtain access to quality programmes, the translation of programmes that have been tested seems to be an interesting option. Members of our group have found that the process of translation of the MoodGYM programme from English to Norwegian is highly cost-efficient (Lintvedt et al., 2013a).

Several internet-based programmes have been developed to prevent and treat depression. Four of these have been widely used and evaluated. A meta-review identified 10 studies published between 1999 and February 2011 and compared the programmes MoodGYM, Color Your Life, Overcoming Depression, and Beating the Blues. While there is some evidence for the effectiveness of MoodGYM, Beating the Blues and Color Your Life, the evidence is not sufficient to consider any one programme as the best (Foroushani et al., 2011).

\section{Internet used to educate on depression}

For many years Australia has been leading in all research on the use of the internet in prevention and treatment of depression. The Australian authorities have prioritized to educate individuals and the general population on depression. A survey performed in Australia showed that more than half of the people with disabling depression or anxiety never contacted the health-care system, because they thought that they had no need to do so (Andrews \& Carter, 2001). A study in Australia showed that when people seek help for depression, they prefer to consult family, friends, and other individuals in the community, rather than healthcare professionals (Highet et al., 2002). If the relatives are not well informed about depression, this help-seeking behavior may increase the feeling of guilt and hopelessness (Griffiths et al., 2011). It is likely that this behavior is a common phenomenon. Consequently, tools that increase general knowledge of the symptoms and treatment of depression are needed (Katon et al., 1997). The programme ReachOut. com (http://www.reachout.com/) was launched in Australia as a national internetbased mental-education health service for young people. It has been accessed by over 6 million users since its launch in 1998 (Burns et al., 2007). In Norway there 
is no government large scale promotion like ReachOut. Our internet site msh.no (http://msh.no) is one of the small sites that seek to help patients with depression. However, there are other sites that address youths that have health problems: the most notable is "Klaraklok" (http://www.klara-klok.no), where questions regarding psychological health are one of the most frequented groups. Helsenorge.no (http://www.helsenorge.no) is the official site for health information in Norway. In my opinion this site is insufficient and too general.

\section{Prevention of depression}

There is some evidence that internet-based interventions can prevent depression (Calear et al., 2009b; Griffiths \& Christensen, 2006) * Our research group at UiT has shown that an unguided internet-based self-help intervention is effective in reducing symptoms of depression and increasing depression literacy in young adults (Lintvedt et al., 2013b). Considerable methodological problems hamper the evaluation of the effect on prevention.

\section{Internet cognitive behavioural therapy (ICBT)-based treatment of depression}

Internet depression treatment is advantageous for the patient, because it is anonymous, free of stigma, and always accessible (Gega et al., 2004), and hence the treatment may reach people who would otherwise be unreachable (Spek et al., 2007). Most of the evidence seems to confirm that internet treatment works and that a supported internet-based depression treatment is superior to self-help programmes without follow-up (Andersson \& Cuijpers, 2009; Spek et al., 2007). A bit surprisingly, one study found that ICBT is superior to face-to-face therapy (Spek et al., 2007).

Our group has shown that the structure of MoodGYM renders it efficient, to use in conjunction with short follow-up consultations in between each module, and that user satisfaction is good (Hoifodt et al., 2013). Compared with the usual faceto-face treatment internet-based programmes for the treatment of depression are cost-effective (Hollinghurst et al., 2010; Naversnik \& Mrhar, 2013)*. 
Internet applications can be used in many ways. They can be used alone, with automated reminders and emails, with therapist assistance by email or by telephone or with varying degrees of face-to-face therapy (Spek et al., 2007). Our group showed that unguided use of MoodGYM relieved symptoms of depression in a student population at low costs (Lintvedt et al., 2013b). We have also found that the level of depression is the strongest predictor of the use of MoodGYM among senior high-school students (Vangberg et al., 2012).

Recently, many applications ("apps") have been used in the treatment of depression (http://www.healthline.com/health-slideshow/top-depression-iphoneandroid-apps\#1). But, few of these apps have been evaluated and their effects are not known.

\section{Challenges in the field of ICBT}

As mentioned above, the natural history of depression is poorly understood. The placebo effect is enormous in this condition. It is virtually impossible to design randomized-controlled studies that are precisely equal. Some studies have shown that all forms of therapy work equally well (Wampold et al., 2002; Hofmann et al., 2010). Time spent working with the programmes is influenced by factors such as mental agility, cognitive ability and reading aptitude. These factors are likely to be affected by depression, leading to early drop-out (Donkin et al., 2011; Davis \& Addis, 1999).

In the drug treatment of depression, the placebo effect is large and variable, with effects up to $75 \%$ in anti-depressant trials. The placebo effect is probably much larger than $75 \%$ when patients engage themselves in face-to-face therapy, because the ill-defined concepts of human trust and confidence play a major role in all therapy. A further complicating factor is that the placebo effect needs to be distinguished from spontaneous remission, life changes, and mere passing of time (Mora et al., 2011).

The manner in which patients are selected for the randomized-controlled studies, the natural history of the disease and the quality of the contact with therapists all influence the outcome of studies of the effect of treatment. These factors hamper the assessment of the treatment effect (Andrews, 2010). In many studies, patients are invited to participate via media announcements. Even if we know that the depression improves after treatment, or at least during the treatment, patients that have the energy enrol themselves in the studies, may be on the way to rid 
themselves of the depression (Almeida et al., 2008).Consequently, one can wonder whether the people who use the programmes really are those passive, depressed patients who need the programme the most, or whether those who use the programme are already getting better.

With this in mind, it is not surprising that there are some doubts about the effect of computerized cognitive behavioural therapy (CCBT). In the conclusion of their paper, So et al. stated: "Despite a short-term reduction in depression at posttreatment, the effect of long follow-up and the function improvement were not significant, with significantly high drop-out. Considering the risk of bias, our metaanalysis implied that the clinical usefulness of current CCBT for adult depression may need to be re-considered downwards in terms of practical implementation and methodological validity" and "considering the lack of the endurance of effectiveness, functional improvement, and the high drop-out rate, our result inevitably casts doubt on the actual practicability of the current CCBT for depression" (So et al., 2013, p1).

Despite these healthy debates about the effect of the interventions, it is my view that the accumulated knowledge justifies a cautious belief that CCBT and ICBT do in fact work.

\section{Understanding the use of the applications}

In most internet-based programmes, the behaviour of the users can be followed via the number of visits, the number of logons, and sometimes the profile of the persons who log on. In addition, time spent on the programme, completion of tasks and outcome can be studied. This provides information on how the applications are used. Such insight has, to date, been impossible in the conventional treatment of depression.

We know that a greater exposure to web-site content and self-engagement is associated with increased benefit (Christensen et al., 2004a; Christensen et al., 2006)* Therefore, a better understanding of the way that users interact with ICBT may increase the effect of these interventions.

In my view, the most significant problem with this type of intervention is that even if the programmes work, people do not use them. Many studies have attempted to understand why the interventions are not used. 
Attrition is defined as "the gradual wearing down of numbers due to friction" or "the gradual diminishing in numbers due to stress" (http://www.thefreedictionary.com/attrition).

In an interesting and thorough review and analysis of behaviour in relation to e-Health programmes, attrition has been classified into two groups: "non-usage attrition", i.e., the phenomenon of non-usage of a programme, and "drop-out attrition", i.e., the loss of participants to follow-up (Eysenbach, 2005). A different definition has been used in a thorough review performed by Christensen et al. (2009), in which the authors use the term adherence as "the extent to which individuals experience the content of the Internet intervention", and drop-out as "individual(s) who fails to complete the research trial protocol associated with an Internet intervention, and thus does not complete trial assessments". These definitions are not in contrast with each other; rather, they are two attempts to describe and analyse the behaviour of the users of the applications. The definitions illustrate the complexities involved in describing the behaviour of the users of ICBT.

Several studies have analysed the behaviour and characteristics of the users of ICBT and have reported drop-out rates ranging from around $20 \%$ to more than $60 \%$. There is general agreement that the adherence to open-access programmes is poor (Titov, 2011;Christensen et al., 2004b)*. Many studies have shown that increased support yields higher adherence (Eysenbach, 2005). However, the picture is complex because of the natural history of depression (De Graaf et al., 2009; Ahn \& Wampold, 2001).

Members of our group observed very low uptake of, and adherence to (8.5\%), the MoodGYM as a preventive programme among a high-school population (Lillevoll et al., pers. com.). Even when we followed up on a group of highly motivated and selected patients, $40 \%$ of them did not adhere to the programme (Hoifodt et al., 2013). Some of the reasons for dropping out were that the programme was designed for youths. However, as always, it was difficult to ask the patients who dropped out about the reasons for that behavior, as they were unlikely to respond to requests and follow-up questions from our team. In a study performed by members of our group, several non-completers were asked to participate in a qualitative study; however, only three of these individuals agreed to do so. For those persons, one of the major problems was that they could not identify with MoodGYM. Moreover, non-completers felt that they needed more time and in-depth analysis of their problems (Wilhelmsen et al., 2013).

Certain factors are associated with high drop-out from ICBT programs in general: time constraints, lack of motivation, technical or computer-access problems, 
depressive episodes or physical illness, lack of face-to-face contact, preference for taking medication, perceived lack of treatment effectiveness, improvement in condition, and burden of the programme (Christensen et al., 2009; Batterham et al., 2008). Eysenbach found that motivation increased with information given before the treatment, ease of enrolment, ease of drop out, usability, "push" factors (reminders, etc.), personal contact (face-to-face or phone interactions), positive feedback, advantages in completing the trial, individual out-of-pocket expenses, workload, competing interventions, external events, peer effect, and experiences (Eysenbach, 2005). Van Dulmen et al. classified motivational factors into technical solutions (such as simplifying doses), behavioural interventions, educational programmes, and social-support interventions (van Dulmen et al., 2007).

Researchers who engaged in qualitative studies have also tried to understand the conditions that are necessary for the efficacy of different ICBT programmes. Gerhards et al. classified adherence factors into three basic types: technical, social (desire for contact, motivation, and belief that the programme was applicable to the attendant's situation), and research-specific factors that may influence the participants (Gerhards et al., 2011). Bendelin et al. classified the 12 persons involved in ICBT into readers, strivers, and doers (Bendelin et al., 2011). Donkin and Glozier pointed out the importance of intrinsic motivators, such as having a sense of control and the ability to identify with the programme (Donkin \& Glozier, 2012). They also pointed out the importance of supporting others, the engagement of the programme once the user is enrolled, and the values of prompting the participants.

Recently, our group performed two qualitative studies to explore patients' experiences of undergoing ICBT treatment by using MoodGYM. We did in-depth interviews with 14 patients (11 completers and 3 non-completers, 5 men and 9 women, aged 22-61 years) who had taken part in our quantitative study (Hoifodt et al., 2013). Recruitment was continuous until the desired total of 14 interviews was reached. The recruitment procedure was strategic. We aimed to include men and women, younger and older, completers and non-completers. We did two phenomenological-hermeneutical analyses on the same interviews.

When we focused on the dimensions that were considered to be helpful for treatment, we found that the active engagement of the patient, guidance by the therapist and the content of the treatment programme were considered helpful. More specifically three factors in the ICBT were helpful to treat depression. Primarily, patients found that the MoodGYM was a source of new knowledge that provided a structured approach to work with their depression. Secondarily, patients could adapt relevant knowledge from MoodGYM to their situation. Finally, the dialogue 
with the therapist was a trusting relationship in which to share thoughts and feelings and to receive feedback and advice, this relationship assisted the patient in making use of the content of MoodGYM (Lillevoll et al., 2013).

An analysis of motivators of persistence in working with MoodGYM among the same 14 patients revealed that a hope of recovery and a desire to gain control of one's life were intrinsic motivators. Also to be able to choose freely how, when, and where to complete the ICBT modules satisfied the participants' need for autonomy. A sense of belonging towards partners, friends, or family was essential for motivation. To identify with ICBT content was also important. Finally, the combination of the experience of connectedness with the acknowledgement, flexibility, and feedback provided by a qualified therapist in face-to-face consultations was an important motivator (Wilhelmsen et al., 2013). Thus, our research has increased the understanding of some of the factors that are important for the creation of the technical and social conditions that are necessary to increase adherence to ICBT.

\section{Future developments}

To improve adherence users need to be motivated by the correct introduction to the program and to understand the need to work actively with the program, also patients must be advised that the involvement of friends or relatives increase their chance of getting benefit from the program. Programmes or therapists need to encourage and to give advice, as well as to induce trust and hope. The technical solutions must be easy to access and to understand. Finally, the degree of contact or automated feedback should be considered.

Nearly all textbooks on CBT emphasize the importance of the patient-therapist alliance for the success of the therapy. However, participants in ICBT trials tend to rate alliance with the program as high. This raises questions regarding whether therapist factors really are as important for a good outcome as has been stated in the psychotherapy literature (Andersson et al., 2012).

We may use the present knowledge to improve the use of, and adherence to, the programmes. The recommendation of these programmes by authorities will promote their use (van Dulmen et al., 2007;Van Voorhees et al., 2013)*. Therefore, we can incentivize general practitioners (GPs) to recommend programmes. Involvement can also be achieved by intermittent motivational face-to-face consultations (Hoifodt et al., 2013). 
Tailoring the programmes (i.e., adapting the programme to the user preferences or social variables entered before or during the use of the programme) has been used to increase adherence (Johansson et al., 2012). Tailoring can also be used to send the patients automated-but personal-electronic reminders to encourage them to complete the programmes. It is technically feasible to use avatars that are programmed to act in certain ways, depending on the variables entered by the user. At Norwegian Centre for Integrated Care and Telemedicine (NST), we have discussed whether experiences from the gaming industry may be used to reduce attrition. A reward system that is activated during the programme and a more exciting interaction with the programmes may increase adherence. The effect on motivation of charging a fee to motivate the use of ICBT, may also increase adherence as the use is then representing a value. However, it may hamper recruitment, as only motivated patients will pay for the programmes.

\section{Integration of ICBT in a stepped-care model}

The UK-based National Institute of Health and Clinical Excellence (NICE) has recommended that CCBT should be used as the first treatment for light to moderate depression. If the depression is serious or the patient do not get better by using CCBT the patient may get closer follow up with normal face to face therapy or may even be admitted to psychiatric hospital. This stepped care approach where the first step is ICPT or CCBT is also part of the Norwegian and Swedish National guidelines.

We performed a review of research on the effectiveness of CBT for depression and anxiety disorders delivered in primary care by primary-care therapists. We found that these programmes were potentially more effective than was usual care and could be delivered by different types of staff in primary care (Hoifodt et al., 2011).

In the earlier part of our studies, it was challenging for patients to use the programmes without any type of follow-up (Vangberg et al., 2012). Therefore, our idea was that if GPs were to follow up use MoodGYM with short motivational consultations, many more depressed patients would be exposed to the programme early in their depression and could recover before serious social costs of the disease had been incurred. In a randomly controlled study (RCT) carried out by psychologists using 106 patients referred by GPs to a university clinic, we showed that short motivational consultations of $20 \mathrm{~min}$ and email reminders inserted between the five 
modules of MoodGYM reduced depression and yielded a relatively low drop-out rate (Hoifodt et al., 2013).

In Australia, Hickie et al. showed that the use of MoodGYM in general practice was difficult, because of monetary and administrative constraints (Hickie et al., 2010). Based on our study (Hoifodt et al., 2013), we also tried to engage GPs to use MoodGYM; however, this task was more difficult than expected. These findings highlight the importance of understanding the setting in which these applications have to be used. We are currently conducting a study with the aim of understanding the reasons for these difficulties.

As a new and very exciting development, the EU has attributed a considerable grant to a project aimed at applying ICBT and CCBT on a large scale. Under the Competitiveness and Innovation Framework Programme (CIP), the CIP Mastermind research programme will be carried out in Denmark, Norway, the UK, Germany, Belgium, Spain, Italy, the Netherlands, Estonia, Turkey and Belgium. It is the first large-scale project focusing on the treatment of depression using CCBT and ICBT. The NST will be the institution responsible for the project in Norway.

\section{Conclusions}

ICBT has the potential to become a major tool for increasing the mental well-being of large sections of the world's populations. ICBT can be used as a tool to raise awareness and knowledge of the symptoms and treatment of depression among the general population. ICBT can be used alone to treat depression or as part of a stepped-care model that can be integrated into the health-care system. It can also be used to prevent recurrence of new episodes of depression. The treatment can be delivered entirely via self-help or with automated motivational feedback messages, automated reminders, reminders written by therapists, and a combination of face-to-face consultations to be held between programme-directed activities. The effect of the progammes increase to some extend with the level of follow up, but the level of follow up to achieve acceptable results is still a matter of debate. Based on a visionary policy, Australia is at the forefront of this research.

However, research in this area is difficult. To obtain a full effect from the technology that is available, we need to improve our understanding of human behaviour in the context of ICBT. Understanding attrition is part of this broader knowledge of human behaviour in relation to ICBT. We also need to learn more about the methods that can be used to apply the technology to the administrative and 
social structures of our society. The EU programme CIP Mastermind is one step in this direction.

To gain knowledge of the interaction between internet-based programmes for the treatment and prevention of depression and humans, we need to engage in research using a broad approach. This research should involve software engineers, anthropologists, sociologists, economists, psychologists, and other medical personnel.

\section{Noter}

${ }^{1}$ Our research group includes Professors Martin Eiseman, Knut Waterloo and, initially, Prof Catharina E. Wang, PhD and senior researcher Mette Bech Risør, PhDs Ove K Lintvedt, Hans Christian Vangberg and Kjersti Lillevoll and PhD students Ragnhild Høifødt and Maja Wilhelmsen

2 *References have been removed to increase readability. The full reference list can be obtained from the author.

\section{Referencer}

Ahn, H.N., \& Wampold, B. E. (2001). Where oh where are the specific ingredients? A metaanalysis of component studies in counseling and psychotherapy. Journal of Counseling Psychology, 48(3), 251-257. doi: 10.1037/0022-0167.48.3.251.

Almeida, L., Kashdan, T.B., Nunes, T., Coelho, R., Albino-Teixeira, A., \& Soares-da-Silva, P. (2008). Who volunteers for phase I clinical trials? Influences of anxiety, social anxiety and depressive symptoms on self-selection and the reporting of adverse events. Eur J Clin Pharmacol, 64, 575-582. doi:10.1007/s00228-008-0468-8

Andersson, G., \& Cuijpers, P. (2009). Internet-based and other computerized psychological treatments for adult depression: a meta-analysis. Cognitive behaviour therapy, 38, 196-205. doi: 10.1080/16506070903318960

Andersson, G., Paxling, B., Wiwe, M., Vernmark, K., Felix, C.B., Lundborg, L., et al. (2012). Therapeutic alliance in guided internet-delivered cognitive behavioural treatment of depression, generalized anxiety disorder and social anxiety disorder. Behav Res Ther, 50, 544-550. http://dx.doi.org/10.1016/j.brat.2012.05.003

Andersson, L.M., Schierenbeck, I., Strumpher, J., Krantz, G., Topper, K., Backman, G., et al. (2013). Help-seeking behaviour, barriers to care and experiences of care among persons with depression in Eastern Cape, South Africa. J Affect Disord. 151(2), 439-448. http:// dx.doi.org/10.1016/j.jad.2013.06.022

Andrews, G. (2001). Should depression be managed as a chronic disease? BMJ, 322, 419-421. doi: PMC1119639/. 
Andrews, G. (2010). Utility of computerised cognitive-behavioural therapy for depression. Br J Psychiatry, 196, 257-258. doi: 10.1192/bjp.bp.109.076356.

Andrews, G., \& Carter, G.L. (2001). What people say about their general practitioners' treatment of anxiety and depression. Med J Aust, 175 Suppl, S48-51.

Barak, A., Klein, B., \& Proudfoot, J.G. (2009). Defining internet-supported therapeutic interventions. Ann Behav Med, 38, 4-17. doi:10.1007/s12160-009-9130-7

Batterham, P.J., Neil, A.L., Bennett, K., Griffiths, K.M., \& Christensen, H. (2008). Predictors of adherence among community users of a cognitive behavior therapy website. Patient Prefer Adherence, 2, 97-105. doi: 10.2196/jmir.1050

Bendelin, N., Hesser, H., Dahl, J., Carlbring, P., Nelson, K.Z., \& Andersson, G. (2011). Experiences of guided Internet-based cognitive-behavioural treatment for depression: a qualitative study. BMC psychiatry, 11, 107. doi:10.1186/1471-244X-11-107

Burns, J., Morey, C., Lagelee, A., Mackenzie, A., \& Nicholas, J. (2007). Reach Out! Innovation in service delivery. Med J Aust, 187, S31-34.

Butler, A.C., Chapman, J.E., Forman, E.M., \& Beck, A.T. (2006). The empirical status of cognitive-behavioral therapy: a review of meta-analyses. Clin Psychol Rev, 26, 17-31.

Christensen, H., Griffiths, K.M., \& Farrer, L. (2009). Adherence in internet interventions for anxiety and depression. J Med Internet Res, 11, e13. doi: 10.2196/jmir.1194

Christensen, H., Griffiths, K.M., \& Jorm, A.F. (2004a). Delivering interventions for depression by using the internet: randomised controlled trial. BMJ, 328, 265. doi: http://dx.doi. org/10.1136/bmj.37945.566632.EE

Christensen, H., Griffiths, K.M., \& Korten, A. (2002). Web-based cognitive behavior therapy: analysis of site usage and changes in depression and anxiety scores. J Med Internet Res, 4, e3. doi: 10.2196/jmir.4.1.e3

Christensen, H., Griffiths, K.M., Korten, A.E., Brittliffe, K., \& Groves, C. (2004b). A comparison of changes in anxiety and depression symptoms of spontaneous users and trial participants of a cognitive behavior therapy website. J Med Internet Res, 6, e46. doi: 10.2196/jmir.6.4.e46

Christensen, H., Griffiths, K.M., Mackinnon, A.J., \& Brittliffe, K. (2006). Online randomized controlled trial of brief and full cognitive behaviour therapy for depression. Psychol Med, 36, 1737-1746.

Collins, K.A., Westra, H.A., Dozois, D.J., \& Burns, D.D. (2004). Gaps in accessing treatment for anxiety and depression: challenges for the delivery of care. Clin Psychol Rev, 24, 583-616.

Cuijpers, P., van Straten, A., van Oppen, P., \& Andersson, G. (2008a). Are psychological and pharmacologic interventions equally effective in the treatment of adult depressive disorders? A meta-analysis of comparative studies. J Clin Psychiatry, 69, 1675-1685. doi: 10.4088/JCP.v69n1102.uiz 1839-1641.

Cuijpers, P., van Straten, A., Warmerdam, L., \& Andersson, G. (2008b). Psychological treatment of depression: a meta-analytic database of randomized studies. BMC psychiatry, 8, 36. doi:10.1186/1471-244X-8-36

Davis, M.J., \& Addis, M.E. (1999). Predictors of attrition from behavioral medicine treatments. Annals of Behavioral Medicine, 21, 339-349. https://www.clarku.us/faculty/addis/ VITAE_files/Predictors\%20of\%20Attrition.pdf

De Graaf, L., Gerhards, S., Arntz, A., Riper, H., Metsemakers, J., Evers, S., et al. (2009). Clinical effectiveness of online computerised cognitive-behavioural therapy without sup- 
port for depression in primary care: randomised trial. The British Journal of Psychiatry, 195, 73-80. doi: 10.1192/bjp.bp.108.054429

Donkin, L., Christensen, H., Naismith, S.L., Neal, B., Hickie, I.B., \& Glozier, N. (2011). A systematic review of the impact of adherence on the effectiveness of e-therapies. J Med Internet Res, 13. doi: 10.2196/jmir.1772

Donkin, L., \& Glozier, N. (2012). Motivators and motivations to persist with online psychological interventions: a qualitative study of treatment completers. J Med Internet Res, 14, e91. doi: 10.2196/jmir.2100

Ebmeier, K.P., Donaghey, C., \& Steele, J.D. (2006). Recent developments and current controversies in depression. The Lancet, 367, 153-167. http://www.neuroscience.ubc.ca/CourseMat/EbmeierKP.pdf

Edejer, T.T.-T. (2000). Disseminating health information in developing countries: the role of the internet. BMJ: British Medical Journal, 321, 797.

Eysenbach, G. (2005). The law of attrition. J Med Internet Res, 7, e11. doi: 10.2196/jmir.7.1.e11

Farrer, L., Christensen, H., Griffiths, K.M., \& Mackinnon, A. (2012). Web-based cognitive behavior therapy for depression with and without telephone tracking in a national helpline: secondary outcomes from a randomized controlled trial. J Med Internet Res, 14, e68. doi: 10.2196/jmir.1859

Foroushani, P.S., Schneider, J., \& Assareh, N. (2011). Meta-review of the effectiveness of computerised CBT in treating depression. BMC psychiatry, 11, 131. doi:10.1186/1471244X-11-131

Gega, L., Marks, I., \& Mataix-Cols, D. (2004). Computer-aided CBT self-help for anxiety and depressive disorders: experience of a London clinic and future directions. J Clin Psychol, 60, 147-157.

Gerhards, S.A., Abma, T.A., Arntz, A., de Graaf, L.E., Evers, S.M., Huibers, M.J., et al. (2011). Improving adherence and effectiveness of computerised cognitive behavioural therapy without support for depression: a qualitative study on patient experiences. J Affect Disord, 129, 117-125.

Godlee, F., Pakenham-Walsh, N., Ncayiyana, D., Cohen, B., \& Packer, A. (2004). Can we achieve health information for all by 2015? The Lancet, 364, 295-300. doi:10.1186/1471244X-11-131

Griffiths, K.M., \& Christensen, H. (2006). Review of randomised controlled trials of Internet interventions for mental disorders and related conditions. Clinical Psychologist, 10, 16-29. doi: 10.1080/13284200500378696

Griffiths, K.M., Crisp, D.A., Barney, L., \& Reid, R. (2011). Seeking help for depression from family and friends: a qualitative analysis of perceived advantages and disadvantages. BMC psychiatry, 11, 196. doi:10.1186/1471-244X-11-196

Hickie, I.B., Davenport, T.A., Luscombe, G.M., Moore, M., Griffiths, K.M., \& Christensen, H. (2010). Practitioner-supported delivery of internet-based cognitive behaviour therapy: evaluation of the feasibility of conducting a cluster randomised trial. Med J Aust, 192, S31-35. https://www.mja.com.au/journal/2010/192/11/practitioner-supported-deliveryinternet-based-cognitive-behaviour-therapy

Highet, N.J., Hickie, I.B., \& Davenport, T.A. (2002). Monitoring awareness of and attitudes to depression in Australia. Med J Aust, 176 Suppl, S63-68. 
Hofmann, S.G., Sawyer, A.T., \& Fang, A. (2010). The empirical status of the »new wave« of cognitive behavioral therapy. Psychiatr Clin North Am, 33, 701-710. doi: 10.1016/j. psc.2010.04.006

Hoifodt, R.S., Lillevoll, K.R., Griffiths, K.M., Wilsgaard, T., Eisemann, M., Waterloo, K., et al. (2013). The clinical effectiveness of web-based cognitive behavioral therapy with face-to-face therapist support for depressed primary care patients: randomized controlled trial. J Med Internet Res, 15, e153. doi: 10.2196/jmir.2714

Hoifodt, R.S., Strom, C., Kolstrup, N., Eisemann, M., \& Waterloo, K. (2011). Effectiveness of cognitive behavioural therapy in primary health care: a review. Fam Pract, 28, 489-504. doi:10.1093/fampra/cmr017

Hollinghurst, S., Peters, T.J., Kaur, S., Wiles, N., Lewis, G., \& Kessler, D. (2010). Cost-effectiveness of therapist-delivered online cognitive-behavioural therapy for depression: randomised controlled trial. Br J Psychiatry, 197, 297-304. doi: 10.1192/bjp.bp.109.073080

Johansson, R., Sjöberg, E., Sjögren, M., Johnsson, E., Carlbring, P., Andersson, T., et al. (2012). Tailored vs. standardized internet-based cognitive behavior therapy for depression and comorbid symptoms: a randomized controlled trial. PLoS ONE, 7, e36905.doi: 10.1371/journal.pone.0036905

Katon, W., Von Korff, M., Lin, E., Unutzer, J., Simon, G., Walker, E., et al. (1997). Populationbased care of depression: effective disease management strategies to decrease prevalence. Gen Hosp Psychiatry, 19, 169-178.

Kessler, R.C., \& Bromet, E.J. (2013). The epidemiology of depression across cultures. Annu Rev Public Health, 34, 119-138. doi: 10.1146/annurev-publhealth-031912-114409

Kleine-Budde, K., Muller, R., Kawohl, W., Bramesfeld, A., Moock, J., \& Rossler, W. (2013). The cost of depression - a cost analysis from a large database. J Affect Disord, 147, 137143. doi: 10.1016/j.jad.2012.10.024

Lillevoll, K.R., Wilhelmsen, M., Kolstrup, N., Hoifodt, R.S., Waterloo, K., Eisemann, M., et al. (2013). Patients' experiences of helpfulness in guided internet-based treatment for depression: qualitative study of integrated therapeutic dimensions. J Med Internet Res, 15, e126. doi:10.1186/1471-244X-13-296

Lintvedt, O.K., Griffiths, K.M., Eisemann, M., \& Waterloo, K. (2013a). Evaluating the translation process of an Internet-based self-help intervention for prevention of depression: a cost-effectiveness analysis. J Med Internet Res, 15(1): e18 doi: 10.2196/jmir.2422.

Lintvedt, O.K., Griffiths, K.M., Sorensen, K., Ostvik, A.R., Wang, C.E., Eisemann, M., et al. (2013b). Evaluating the effectiveness and efficacy of unguided internet-based self-help intervention for the prevention of depression: a randomized controlled trial. Clin Psychol Psychother, 20, 10-27. doi: 10.1002/cpp.770

Mathers, C.D., \& Loncar, D. (2006). Projections of global mortality and burden of disease from 2002 to 2030. PLoS medicine, 3, e442. doi: 10.1371/journal.pmed.0030442

Mora, M.S., Nestoriuc, Y., \& Rief, W. (2011). Lessons learned from placebo groups in antidepressant trials. Philos Trans R Soc Lond B Biol Sci, 366, 1879-1888.

Mørch, M.M., \& Rosenberg, N.K. (2005). Kognitiv terapi: modeller og metoder (Cognitive therapy: models and methods): Hans Reitzel, Copenhagen.

Naversnik, K., \& Mrhar, A. (2013). Cost-effectiveness of a novel e-health depression service. Telemed J E Health, 19, 110-116. 
Patel, V., Araya, R., Chatterjee, S., Chisholm, D., Cohen, A., De Silva, M., et al. (2007). Treatment and prevention of mental disorders in low-income and middle-income countries. The Lancet, 370, 991-1005. doi: 10.1016/S0140-6736(07)61240-9

Powell, J., Hamborg, T., Stallard, N., Burls, A., McSorley, J., Bennett, K., et al. (2013). Effectiveness of a web-based cognitive-behavioral tool to improve mental well-being in the general population: randomized controlled trial. J Med Internet Res, 15, e2. doi: 10.2196/ jmir.2240

Prukkanone, B., Vos, T., Bertram, M., \& Lim, S. (2012). Cost-effectiveness analysis for antidepressants and cognitive behavioral therapy for major depression in Thailand. Value Health, 15, S3-8. doi: 10.1016/j.jval.2011.11.009

Reza, A., Mercy, J.A., \& Krug, E. (2001). Epidemiology of violent deaths in the world. Injury Prevention, 7, 104-111. doi: 10.1136/ip.7.2.104.

Rhebergen, D., Beekman, A.T., Graaf, R., Nolen, W.A., Spijker, J., Hoogendijk, W.J., et al. (2009). The three-year naturalistic course of major depressive disorder, dysthymic disorder and double depression. J Affect Disord, 115, 450-459. doi: 10.1016/j.jad.2008.10.018

Sado, M., Yamauchi, K., Kawakami, N., Ono, Y., Furukawa, T.A., Tsuchiya, M., et al. (2011). Cost of depression among adults in Japan in 2005. Psychiatry Clin Neurosci, 65, 442-450. doi: 10.1111/j.1440-1819.2011.02237.x

So, M., Yamaguchi, S., Hashimoto, S., Sado, M., Furukawa, T.A., \& McCrone, P. (2013). Is computerised CBT really helpful for adult depression?-A meta-analytic re-evaluation of CCBT for adult depression in terms of clinical implementation and methodological validity. BMC psychiatry, 13, 113. http://www.biomedcentral.com/1471-244X/13/113

Spek, V., Cuijpers, P., Nyklicek, I., Riper, H., Keyzer, J., \& Pop, V. (2007). Internet-based cognitive behaviour therapy for symptoms of depression and anxiety: a meta-analysis. Psychol Med, 37, 319-328. doi: 10.1017/S0033291706008944

Titov, N. (2011). Internet-delivered psychotherapy for depression in adults. Curr Opin Psychiatry, 24, 18-23. doi: 10.1097/YCO.0b013e32833ed18f.

van Dulmen, S., Sluijs, E., van Dijk, L., de Ridder, D., Heerdink, R., \& Bensing, J. (2007). Patient adherence to medical treatment: a review of reviews. BMC health services research, 7, 55. doi: 10.1186/1472-6963-7-55

Van Voorhees, B.W., Hsiung, R.C., Marko-Holguin, M., Houston, T.K., Fogel, J., Lee, R., et al. (2013). Internal versus external motivation in referral of primary care patients with depression to an internet support group: randomized controlled trial. J Med Internet Res, 15, e42. http://www.ncbi.nlm.nih.gov/pmc/articles/PMC3636270/

Vangberg, H.C., Lillevoll, K.R., Waterloo, K., \& Eisemann, M. (2012). Does Personality Predict Depression and Use of an Internet-Based Intervention for Depression among Adolescents? Depress Res Treat, 2012, 593068. doi: 10.1155/2012/593068

Wampold, B.E., Minami, T., Baskin, T.W., \& Callen Tierney, S. (2002). A meta-(re)analysis of the effects of cognitive therapy versus 'other therapies' for depression. J Affect Disord, 68, 159-165. http://dm.education.wisc.edu/tminami/intellcont/Wampold_etal_ JAD_2002-1.pdf

Wells, K.B., Burnam, M.A., Rogers, W., Hays, R., \& Camp, P. (1992). The course of depression in adult outpatients. Results from the Medical Outcomes Study. Arch Gen Psychiatry, 49, 788-794. http://homepage.psy.utexas.edu/HomePage/Class/Psy394Q/Behavior\%20Therapy\%20Class/Assigned\%20Readings/Depression\%20Lecture/Wells92.pdf 
Wilhelmsen, M., Lillevoll, K., Risør, M.B., Høifødt, R., Johansen, M.-L., Waterloo, K., et al. (2013). Motivation to persist with internet-based cognitive behavioural treatment using blended care: a qualitative study. BMC psychiatry, 13, 296. doi: 10.1186/1471-244X-13-296 